\title{
Psychosocial Aspects of Rheumatic Diseases: Introduction
}

\author{
Erik Taal, MA, Erwin R. Seydel, PhD, Johannes J. Rasker, MD \\ and Oene Wiegman, PhD
}

Department of Psychology, University of Twente, P.O. Box 217, 7500 AE Enschede (Netherlands)

\section{Introduction}

This special issue of Patient Education and Counseling deals with psychosocial and patient-educational aspects of rheumatic diseases, comprising more than 100 chronic diseases with complaints involving the joints and/or connective tissues. The most prevalent forms of rheumatic diseases are osteoarthritis, rheumatoid arthritis (RA), ankylosing spondylitis and fibromyalgia. The causes of many rheumatic conditions are unknown, and these diseases cannot be prevented or completely cured. However, due to the proportional increase of the ageing population, the number of patients with rheumatic diseases is growing rapidly (Fielts and Yelin, 1989).

\section{Disease Characteristics}

Rheumatoid arthritis is a chronic, disabling disease characterized by inflammation of joints and joint tissues. This inflammation may be remitting, but if continued often results in progressive joint destruction, deformity and ultimately in various degrees of incapacitation (Rasker and Cosh, 1987). Cardinal symptoms are persistent pain, stiffness and swelling in joints. More general complaints are morning stiffness and fatigue, sometimes due to anaemia. The course of RA is chronic and unpredictable. Patients experience periods of exacerbation and remission of disease activity (Schumacher, 1988; Rasker and Cosh, 1987). The activity of the disease can vary greatly, sometimes even daily. RA can strike people of all ages but mostly becomes manifest between the ages of 20 and 50. Women are more affected than men; the overall ratio is 3:1 (Anderson et al., 1985). It is estimated that the prevalence of RA in most white populations approaches $1 \%$ among adults aged 18 and older (Schumacher, 1988).

Osteoarthritis is characterized by progressive loss of articular cartilage and secondary reactions in bone. Important clinical features are joint pain, stiffness at the start of movements and bony enlargement with limitation of motion. Prevalence of osteoarthritis increases with age; the disease is almost universal in people 65 years or older (Schumacher, 1988).

Ankylosing spondylitis is a chronic, progressive disease predominantly affecting the spine. Major symptoms are back pain and stiffness of the spine with reduced mobility. The disease usually starts before 40 years of age and is predominantly seen in men (Schumacher, 1988).

Fibromyalgia is a nonarticular disease characterized by widespread musculoskeletal pain, stiffness, disturbed sleep and fatigue. There is a lack of objective findings. The disease occurs predominantly $(80-90 \%)$ in women usually in the age group between 20 and 50 years (Schumacher, 1988; Yunus et al., 1988). 


\section{Psychosocial Aspects of Rheumatic Diseases}

Rheumatic diseases have a great impact on the quality of life. They affect not only physical functioning but also psychological and social aspects (Anderson et al., 1985). Rheumatic diseases have on the one hand behavioral, psychological and social consequences, but on the other hand behavioral, psychological and social variables may be determinants of disease development and of patients' ability to adapt to their condition.

Research on psychosocial aspects of rheumatic diseases focuses on four major areas: (1) the role of behavioral and psychosocial factors in the onset and course of rheumatic disease; (2) the psychosocial and behavioral consequences of rheumatic disease; (3) the mediating influence of psychosocial factors on patients' ability to adjust to the consequences of their disease; and (4) the development of psychosocial treatment strategies, e.g. models of patient education.

Next to these four major areas social scientists, and other researchers, are also involved in the development of methods to assess physical and psychosocial aspects of the health status of arthritis patients. To investigate the role of physical, behavioral, psychological and social variables in rheumatic diseases and to assess the effects of medical and psychosocial treatment programs reliable and validated measurement instruments are needed. In the last decade several instruments have been developed to assess the physical, psychological and social aspects of the health status of arthritis patients (Anderson et al., 1985). An important methodological problem in the assessment of the psychological impact of rheumatic disease is that many instruments like for instance the MMPI or the BDI were originally validated with psychiatric patients (Bradley, 1989b). Depression scales may overestimate the prevalence and severity of depression among persons with arthritis due to the endorsement of items such as "I felt everything I did was an effort" or "I am about as able to work as I ever was" (Blalock et al.,
1989; Pincus et al., 1986). Questions about physical symptoms may be accurate to assess emotional distress among psychiatric patients but in arthritis patients these questions measure symptoms that reflect their actual physical condition.

(1) The role of psychosocial factors in the onset and course of rheumatic disease

There has been much research into the role of psychosocial factors in the onset and course of rheumatic diseases, especially RA. Already in the start of the century it was suggested that certain personality typologies predisposed to get rheumatoid arthritis (Jones, 1909). Studies into this so called specificity hypothesis have led to conflicting results. More recent research suggests that the observed psychological characteristics in RA patients may be reactions to the physical disease process rather than causal factors (Anderson et al., 1985; Lerman, 1987).

In the scientific literature, however, indications can be found that stressful lifeexperiences modulate the onset and course of RA (Anderson et al., 1987; Lerman, 1987). The mechanisms through which stress exerts its influence on the disease process are still unclear. The effects of stress on the onset and course of RA seem to be determined for a great deal by the type of strategies people use to cope with stressful experiences (Wegener, 1991). Recent developments indicate that stress influences the immunc system (Bradley, 1989b; Wegener, 1991). Zautra et al. (1989) found indications that stress may lead to negative reactions in the immune system of RA patients. However, this study showed no consistent relationships between stress and changes in disease activity. Future research in psychoneuroimmunology may lead to a better understanding of the complex relationships between stress, immune system functioning and the onset and course of RA.

\section{(2) Psychosocial and behavioral consequences of rheumatic disease}

For the rheumatic patient, it is probably less significant that stress moderates the onset 
or course of his or her disease than it is that a rheumatic disease with an often unpredictable and painful course has a stressful impact on the life of the patients. The rheumatic disease will have physical as well as behavioral and psychosocial implications for the patient and his or her family. The quality of life of rheumatic patients can be characterized by functional disability (e.g. restrictions in mobility and daily activities), pain, loss of independence, psychological problems like depression and anxiety, changes in family functioning and social activities, work disability and financial problems (Anderson et al., 1985; Cornelissen et al., 1988). Also the adherence to medication and other therapies can be problematic to the patient (Bradley, 1989a).

\section{(3) Mediating psychosocial factors}

There is accumulating evidence that the behavioral and psychosocial impact is not directly related to the severity of the rheumatic disease, but that there is considerable individual variation in adjustment to the disease. Psychosocial factors can have a mediating influence on the patients' ability to adjust to the consequences of their disease (Bradley, 1989b; Wegener, 1991). McFarlane and Brooks (1988) found that psychological factors, like denial of emotional distress or depression, predicted more of the variance in disability, over a 3-year period, than disease activity in 30 RA patients. Several studies have demonstrated relationships between the use of certain coping strategies and adjustment (Bradley, 1989b). RA patients' use of negative coping strategies such as catastrophizing, escapist fantasies and engaging in passive pain management strategies is associated with poor psychological status and high levels of functional impairment (Brown et al., 1989; Keefe et al., 1989; Revenson and Felton, 1989). Various studies have indicated that cognitive factors like self-efficacy or learned helplessness are related to functional and psychological impairment (Lorig et al., 1989; Nicassio et al., 1985). The unpredictable nature of RA and the varying disease activity may cause patients to view their disease as uncontrollable. This often leads to low selfefficacy expectations about needed causes of action to cope with the consequences of the disease or to more generalized feelings of learned helplessness; that is the experience of having no control over one's life, in general, and across different situations. Social support from e.g. family and friends can also play a significant role in the adaptation to the disease. Significant relationships have been found between social support and health status in arthritis patients (Affleck et al., 1988; Weinberger et al., 1990; Goodenow et al., 1990).

A substantial problem is the often low rate of adherence to treatment regimens which is greatly influenced by behavioral and psychosocial factors. Determining factors are the doctor-patient relationship, knowledge about the nature of the regimen, expectations about the efficacy of the treatment, skills and selfefficacy expectations (Bradley, 1989a; Stewart Agras, 1989). Furthermore, social support can motivate patients to adhere to treatment recommendations.

\section{(4) Psychosocial interventions}

Because of the moderating role of behavioral and psychosocial factors in rheumatic diseases, and the limited possibilities of medical interventions, there has been an increased interest in psychosocial interventions like patient education or psychotherapy from behavioral scientists and health professionals such as physicians, nurses, and others. They want patients to become more informed about their conditions, to improve adherence to therapeutic regimens, learn self-management strategies for effectively coping with the demands of the disease, and to prevent disability. Psychosocial interventions should not be limited to the transfer of knowledge but should be aimed at the teaching of selfmanagement skills that are useful for an adequate handling of the consequences of the disease. When studying the effects of psycho- 
social interventions one should not only measure changes in knowledge but also changes in behavior and physical and psychosocial health status. In recent years various reviews have been published about the effectiveness of psychosocial interventions (Lorig et al., 1987; McCracken, 1991; Mullen et al., 1987; Kirwan, 1990). These reviews indicate that psychosocial interventions can be effective in changing knowledge, behaviors, and physical and psychosocial health status. Furthermore, it is concluded that future studies should investigate relevant factors that can facilitate or mediate beneficial effects, thereby clarifying the causal relation between intervention and outcome (Lorig et al., 1987; McCracken, 1991; Kirwan, 1990). This conclusion points at the importance of thorough analysis of the health problems and determinants of these problems before designing an intervention (Green and Kreuter, 1991). This means that there has to be a careful planning of the intervention (Green and Kreuter, 1991; Kok, 1992). The effectiveness of psychosocial interventions depends heavily on the quality of planning. That means a careful analysis of the problem, the behavior, the determinants, the intervention and implementation and of the strength of the relationship between those aspects. A thorough problem analysis is a sine qua non. Few physicians would think of prescribing medication without first diagnosing the probable cause of the illness. Nevertheless, the same physicians, when confronted with the problem of behavior change, frequently do not realize that the determinants of behavior change are multiple and complex. Instead, when confronted with patient nonadherence, they tend to assume that the patient either does not understand the instructions or is not motivated to comply. In any case they are convinced that they have done their work, namely giving patient education and information. They apparently do not realize that they have to give patient education in a systematic way and that knowledge and motivation are only two of many variables that can influence behaviour.

\section{Contents of this Special Issue}

On June 6-9 1990 the third International Symposium for Health Professionals in Rheumatology was held in Enschede, the Netherlands. Some of the major themes at the symposium were psychosocial aspects of rheumatic diseases, patient education and assessment methods. The articles in this special issue of Patient Education and Counseling are mainly based on presentations at the symposium. Without pretending to be exhaustive they give an impression of the topics dealt with at the symposium. The contributions can be subdivided into three sections. The first section of the issue contains articles concerning the various physical, behavioral and psychosocial problems of patients with rheumatic disease as well as the role of behavioral and psychosocial factors in the process of adjustment to the demands of the disease. The articles in this part may provide leads for the development of psychosocial interventions. The second section contains articles on methods of assessing the physical and psychosocial aspects of health status. When assessing the problems of rheumatic patients or evaluating the efficacy of medical or psychosocial treatments it is necessary to have reliable and valid means to assess health status. The final section of the issue contains articles that discuss the development and evaluation of psychosocial intervention programs.

In the first section Taal et al. describe a Dutch study on patients with rheumatoid arthritis. The health problems of RA patients are assessed, the problems these patients experience in adhering to health recommendations and the relationships of these problems with self-efficacy expectations and social support. Implications for the development of a patient education program are discussed.

Samuelsson et al. discuss the problems of rheumatic patients in the early stages of treatment and their needs and expectations regarding psychosocial care. Some results of their Swedish studies are presented on the psycho- 
social needs and expectations of rheumatic patients before their first visit to a doctor at the outpatient specialist clinics. Implications of the studies are discussed for practical clinical work and psychosocial care at an early stage of the disease.

Eberhard et al. report a Swedish longitudinal study on the interaction of RA and psychological factors over two years in a group of 89 patients with newly established disease. A newly developed psychological adjustment test is applied and validated. Eberhard et al. conclude from their study that symptoms of psychological distress are in general not very pronounced, fairly stable over time, and not related to disease severity. The patients' degree of adjustment changes slowly or not at all during the two years.

Krol et al. give an introduction on the concepts of social network and social support and describe the possible effects of social support on the quality of life among patients with RA. Subsequently they review studies on social support and rheumatic disease published in the period from 1986 to 1991 . They conclude that patients can experience positive effects form social support, but that further research remains necessary to gain insight in the beneficial elements and mechanisms of social support. Finally they stress the importance of gaining more insight into the personality structure of patients as a possible intervening factor.

In the second section on the assessment of health status Jacobs et al. give an overview of existing instruments to assess physical and psychosocial dimensions of health status. Properties of frequently used instruments are described and their validity, reliability and sensitivity to detect change are discussed.

Pincus and Callahan discuss the usc of depression scales in patients with rheumatoid arthritis. Scales to measure depression like the MMPI, BDI or CES-D include statements concerning somatic symptoms like fatigue or pain that are common in patients with a rheu- matic condition but that also may reflect depression. Pincus and Callahan give a summary of evidence for this so called 'criterion contamination' in responses of RA patients on the MMPI, BDI and CES-D from their own studies and studies from other research groups. They conclude that depression appears to be more common in patients with $\mathrm{RA}$ versus the general population, and that depression scales may be used in RA patients, but results must be interpreted with caution, taking into account criterion contamination.

Bakker et al. give an introduction on health related utility measurement in rheumatology. Commonly used instruments to assess health related quality of life, as discussed by Jacobs et al. in this issue, usualiy assess various physical and psychosocial aspects of health. Utility measures are single measures of the value or preference that respondents attach to their overall health status. According to Bakker et al. utility measures can be very useful as a measure of effect in evaluating treatments. They state that utility measures have the advantage that they summarize both negative and positive effects of an intervention into one single value. In their article Bakker et al. discuss the various approaches to utility measurement and describe the Maastricht Utility Measurement Questionnaire as an example of utility measurement. Finally they discuss the concept of Quality Adjusted Life Years (QALYs). A QALY is a single comprehensive outcome measure that includes effects in terms of both quality of life and survival.

In the third section on the development and evaluation of interventions Barlow et al. discuss self-help groups for patients with ankylosing spondylitis as a means of promoting exercise treatment. They present a comparative study of 111 members and 50 non-members of such groups along psychosocial dimensions including health locus of control, social support and health behavior. Although no comparable measurements are available for non-members, the data on a subset of 20 self-help group members suggest 
that the adoption of a regular exercise program can have beneficial effects on physical mobility. Self-help group members are distinguished from non-members by a combination of factors including a low reliance on powerful others health locus of control beliefs, greater satisfaction with available support, and increased frequency of exercise.

Basler describes a German cognitivebehavioral treatment programme in a group setting format for chronic pain patients. In a meta-analysis, treatment effects in different diagnostic groups (low back pain, tension headache, rheumatoid arthritis, ankylosing spondylitis) are compared. Pain reduction is greatest in low back pain and least in ankylosing spondylitis. The effectiveness of the treatment in RA patients and AS patients seems to pertain more to emotional stabilization than to pain reduction.

In the last article of this special issue Taal et al. discuss the development and evaluation of a Dutch group education program for patients with rheumatoid arthritis. This program is based on social learning theory (Bandura, 1986) and the Arthritis Self-Management Course (ASMC) developed in the USA by Lorig (Lorig et al., 1985; Lorig et al., 1989). The ASMC has been shown to be successful and leads to increased knowledge, performance of taught behaviors and less pain. The goal of the program of Taal et al. is the strengthening of self-efficacy, outcome expectations and self-management behaviors which may lead to better health status. Evaluation of the program showed beneficial effects on knowledge, self-efficacy, outcome expectations, behavior, functional disability and joint tenderness.

\section{References}

Affleck, G., Pfeiffer, H., Tennen, J. and Fifield, J. (1988). Social support and psychosocial adjustment to rheumatoid arthritis. Arthritis Care and Research, 1, 71-77.

Anderson, K.O., Bradley, L.A., Young, L.D., McDaniel, L.K. and Wise, C.M. (1985). Rheumatoid Arthritis: review of psychological factors related to etiology, effects, and treatment. Psychological Bulletin, 98, 358-387.

Bandura, A. (1986). Social foundations of thought and action: $A$ social cognitive theory. Englewood: Prentice-Hall.

Blalock, S.J., DeVellis, R.F., Brown, G.K. and Wallston, K.A. (1989). Validity of the center for epidemiological studies depression scale in arthritis populations. Arthritis and Rheumatism, 32, 991-997.

Bradley, L.A. (1989a). Adherence with Treatment Regimens Among Adult Rheumatoid Arthritis Patients: Current Status and Future Directions. Arthritis Care and Research, 2, 33-39.

Bradley, L.A. (1989b). Psychosocial factors and disease outcomes in rheumatoid arthritis: old problems, new solutions, and a future agenda. Arthritis and Rheumatism, 32, 1611-1614.

Brown, G.K., Nicassio, P.M. and Wallston, K.A. (1989). Pain coping strategies and depression in rheumatoid arthritis. Journal of Consulting and Clinical Psychology, 57, 652-657.

Cornelissen, P.G.J., Rasker, J.J. and Valkenburg H.A. (1988). The arthritis sufferer and the community: a comparison of arthritis sufferers in rural and urban areas. Annals of the Rheumatic Diseases, 47, 150-156.

Goodenow, C., Reisine, S.T. and Grady, K.E. (1990). Quality of Social Support and Associated Social and Psychological Functioning in Women With Rheumatoid Arthritis. Health Psychology, 9, 266-284.

Green, L.W. and Kreuter, M.W. (1991). Health promotion planning, an educational and environmental approach. Mountain View, California: Mayfield.

Jones, R.L. (1909). Arthritis deformans. New York: William Wood.

Fielts, W. and Yelin, E. (1989). The economic impact of the rheumatic diseases in the United States. Arthritis and Rheumatism, 33, 750-755.

Keefe, F.J., Brown, G.K., Wallston, K.A. and Caldwell, D.S. (1989). Coping with arthritis pain: Catastrophizing as a maladaptive strategy. Pain, 37, 51-56.

Kirwan, J.R. (1990). Patient education in rheumatology. Current Opinion in Rheumatology, 2, 336-339.

Kok, G. (1992). Quality of planning as a decisive determinant of health education effectiveness. Hygie, International Journal of Health Education, 11 (4), 5-8.

Lerman, C.E. (1987). Rheumatoid arthritis: Psychological factors in the etiology, course and treatment. Clinical Psychology Review, 7, 413-425.

Lorig, K., Chastain, R., Ung, E., Shoor, S. and Holman, H.R. (1989). Development and evaluation of a scale to measure perceived self-efficacy in people with arthritis. Arthritis and Rheumatism, 32, 37-44.

Lorig, K., Konkol, L. and Gonzalez, V. (1987). Arthritis patient education: a review of the literature. Patient Education and Counseling, 10, 207-252.

Lorig, K., Lubeck, D., Kraines, R.G., Seleznick, M. and Holman, H.R. (1985). Outcomes of self-help education for patients with arthritis. Arthritis and Rheumatism, 28, 680-685. 
McCracken, L.M. (1991). Cognitive-behavioral treatment of rheumatoid arthritis: a preliminary review of efficacy and methodology. Annals of Behavioral Medicine, 13, 57-65.

McFarlane, A.C. and Brooks, P.M. (1988). Determinants of disability in rheumatoid arthritis. British Journal of Rheumatology, 27, 7-14.

Mullen, P.D., Laville, E.A., Biddle, A.K. and Lorig, K. (1987). Efficacy of psychoeducational interventions on pain, depression and disability in people with arthritis: a meta-analysis. Journal of Rheumatology, 14, 33-39.

Nicassio, P.M., Wallston, K.A., Callahan, L.F., Herbert, M. and Pincus, $T$ (1985) The measurement of helplessness in rheumatoid arthritis: The development of the arthritis helplessness index. Journal of Rheumatology, 12, 462-467.

Pincus, T., Callahan, L.F., Bradley, L.A., Vaughn, W.K. and Wolfe, F. (1986). Elevated MMPI scores for hypochondriasis, depression, and hysteria in patients with rheumatoid arthritis reflect disease rather than psychological status. Arthritis and Rheumatism, 29, 1456-1466.

Rasker, J.J. and Cosh, J.A. (1987). The natural history of rheumatoid arthritis over 20 years. Clinical symptoms, radiological signs, treatment, mortality and prognostic significance of early features. Clinical Rheumatology, 6 (Suppl. 2), 5-11.

Revenson, T.A. and Felton, B.J. (1989). Disability and coping as predictors of psychological adjustment to rheumatoid arthritis. Journal of Consulting and Clinical Psychology, 57, 344-348.

Schumacher, H.R. (Ed). (1988). Primer on the Rheumatic Diseases, ninth edition, Atlanta GA: Arthritis Foundation.

Stewart Agras, W. (1989). Understanding compliance with the medical regimen: The scope of the problem and a theoretical perspective. Arthritis Care and Research, 2, S2-S7.

Wegener, S.T. (1991). Psychosocial aspects of rheumatic disease: the developing biopsychosocial framework, Current Opinion in Rheumatology, 3, 300-304

Weinberger, M., Tierney, W.M., Booher, P. and Hiner, S.L. (1990). Social support, stress and functional status in patients with osteoarthritis. Social Science and Medicine, 30, 503-508.

Yunus, M.B., Kalyan-Raman, U.P. and Kalyan-Raman, K. (1988). Primary fibromyalgia syndrome and myofascial pain syndrome: Clinical features and muscle pathology. Archives of Physical and Medical Rehabilitation, 69, 451-454.

Zautra, A.J., Okun, M.A., Robinson, S.E., Lee, D., Roth, S.H. and Emmanual, J. (1989). Life stress and lymphocyte alterations among patients with rheumatoid arthritis. Health Psychology, 8, 1-14.

Correspondence to: Erik Taal, MA, Department of Psychology, University of Twente, P.O. Box 217, 7500 AE Enschede, Netherlands. 